\section{Cholesterol-lowering therapy may retard the progression of diabetic nephropathy}

Dear Sir,

Lam et al. [1] investigated the effect of cholesterol-lowering therapy with an $\mathrm{HMG}$ CoA reductase inhibitor (lovastatin) for 2 years on the progression of diabetic nephropathy in 34 non-insulin-dependent diabetic (NIDDM) patients. The authors conclude that effective normalization of hypercholesterolaemia may retard the progression of diabetic nephropathy. I would like to challenge this conclusion, based on the following considerations.

A heterogeneous group of NIDDM patients was investigated, 10 with microproteinuria, and 24 with macroproteinuria. No information is available on the causes of proteinuria. This is important since previous studies have demonstrated that $25-40 \%$ of macroalbuminuric NIDDM patients suffer from non-diabetic glomerulopathies [2-4]. Proteinuric NIDDM patients with no retinopathy have a fifty-fifty chance of having diabetic or non-diabetic glomerulopathy [2].

It is difficult to understand why the investigation was carried out as a single blind rather than a double blind study.

The authors applied intra group test rather than inter group test for their statistical analysis. The former procedure is inappropriate since the correct statistical analysis in a parallel study is to compare the change from baseline to 2 years in the main end-point variables (glomerular filtration rate [GFR], creatinine, proteinuria) between the two groups. No power calculation is presented.

The lovastatin and placebo treated groups both had a rise in proteinuria and serum creatinine while glomerular filtration rate remained unchanged in the actively treated arm and dropped in the placebo treated arm. The drop in the placebo treated arm occurred from 12 to 24 months. However, the authors state: "There was no significant difference in GFR, serum creatinine, or 24-h urinary protein excretion between the two groups at baseline, 12 months or 24 months". This intergroup analysis is more valid than the intragroup analysis mentioned above. However, the correct analysis is comparison of the changes which occurred during the trial period between the two groups.

Finally, the two original studies dealing with the effect of HMG CoA reductase inhibitor in IDDM and NIDDM patients with hypercholesterolaemia and diabetic nephropathy have been neglected $[5,6]$.

The present study does not allow for a valid interpretation regarding the potential beneficial effect of cholesterol-lowering therapy due to flaws in patient material, diagnostic criteria for nephropathy, design and most important incorrect statistical analysis.

Yours sincerely,

H.-H. Parving

\section{References}

1. Lam KS, Cheng IKP, Janus ED, Pang RWC (1995) Cholesterol-lowering therapy may retard the progression of diabetic nephropathy. Diabetologia 38: 604-609

2. Parving H-H, Gall M-A, Skøtt P et al. (1992) Prevalence and causes of albuminuria in non-insulin-dependent diabetic patients. Kidney Int 41: 758-762

3. Gambara V, Mecca G, Remuzzi G, Bertani T (1993) Heterogeneous nature of renal lesions in type II diabetes. J Am Soc Nephrol 3: 1458-1466

4. Lipkin GW, Yeates C, Howie A, Michael J, Adu D, Richards NT (1994) More than one third of type 2 diabetics with renal disease do not have diabetic nephropathy; a prospective study. J Am Soc Nephrol 5: 379 (Abstract)

5. Hommel E, Andersen P, Gall M-A et al. (1992) Plasma lipoproteins and renal function during simvastatin treatment in diabetic nephropathy. Diabetologia 35: 447-451

6. Nielsen S, Schmitz O, Møller N et al. (1993) Renal function and insulin sensitivity during simvastatin treatment in type 2 (non-insulin-dependent) diabetic patients with microalbuminuria. Diabetologia 36: 1079-1086 


\section{Response from the authors}

Dear Sir,

With reference to the comments from Dr. Parving regarding our study published in Diabetologia [1], I wish to offer the following explanations.

Regarding the cause of proteinuria in our patients, 20 $(59 \%)$ had diabetic retinopathy. Among the 14 patients who had no retinopathy only 5 were hypertensive, 2 had hypertension and diabetes diagnosed at the same time and 3 had hypertension diagnosed 3-9 years after the diagnosis of diabetes, around the time of presentation of proteinuria. The other 10 had known diabetes for a mean of 5.3 years. None of the 34 patients had other known causes of parenchymal or obstructive kidney diseases. We can thus assume that the great majority of the study patients had diabetic glomerulopathy.

At any rate, a benefit of cholesterol lowering therapy on renal function has also been reported in patients with non-diabetic nephrotic syndrome [2].

The aim of this study was to compare the effect on renal function of normalization of hypercholesterolaemia vs persistent hypercholesterolaemia, and not simply to compare the effects of lovastatin vs placebo on serum lipids and renal function. Thus the dose of lovastatin had to be increased until the target cholesterol level was achieved. There were therefore logistic difficulties in using the double-blind design. Anyway, although the physician was aware of the grouping, the control of glycaemia and hypertension was very similar between the two groups. Furthermore, the research nurse who carried out the glomerular filtration rate (GFR) studies and the laboratory technicians were not aware of the grouping of the study subjects and hence the conclusions regarding the changes in renal function could not have been affected by the study design.

We applied both intragroup and intergroup statistical tests which are both acceptable means of statistical analysis for these data. Although this study involves more patients and a much longer study period than previous studies addressing the benefit of cholesterol-lowering therapy on diabetic nephropathy $[3,4]$, the patient numbers and study duration were still

Corresponding author: Dr. K.S. L. Lam, Department of Medicine, The University of Hong Kong, Queen Mary Hospital, Hong Kong relatively insufficient for studying the progression of a chronic pathological process, for which the rate of deterioration is highly variable between patients though quite constant for a given individual. The above factors may explain why we could not demonstrate a statistically significant difference in GFR between the two groups at 24 months, although there was a trend towards a lower mean GFR in the placebo group. In the conclusion we have already conceded that our findings were preliminary and only supportive (not confirmative) of a potential benefit of cholesterol-lowering therapy on the progression of nephropathy in these patients.

The two studies referred to by Dr. Parving [3, 4] showed no benefir of simvastatin on renal function in IDDM (12 on treatment) and NIDDM ( 8 on treatment) patients after 12 and 36 weeks, respectively. These negative findings can be readily explained by the small numbers of patients and short study duration as explained above.

In conclusion, we believe that our conclusions, as presented in the published manuscript, are definitely valid. We also wish to thank the many workers in this field who have expressed interest in our preliminary findings. We certainly hope that longterm, multi-centre studies involving much larger numbers of patients can be carried out to address this controversial issue which is of potential therapeutic importance.

Yours sincerely,

K.S.L.Lam

\section{References}

1. Lam KS, Cheng IKP, Janus ED, Pang RWC (1995) Cholesterol-lowering therapy may retard the progression of diabetic nephropathy. Diabetologia 38: 604-609

2. Chan PCK, Robinson JD, Yang WC, Cheng IKP, Yeung HWD, Tsang MTS (1992) Lovastatin in glomerulonephritis patients with hyperlipidaemia and heavy proteinuria. Nephrol Dial Transplant 7: 93-99

3. Hommel E, Andersen P, Gall M-A et al. (1992) Plasma lipoproteins and renal function during simvastatin treatment in diabetic nephropathy. Diabetologia 35: 447-451

4. Nielsen S, Schmitz O, Moller N et al. (1993) Renal function and insulin sensitivity during simvastatin treatment in type 2 (non-insulin-dependent) diabetic patients with microalbuminuria. Diabetologia 36: 1079-1086

\section{The effect of cholesterol-lowering therapy on the progression of diabetic nephropathy is unproved}

\section{Dear Sir,}

In a recent paper Lam et al. [1] asserted that cholesterol-lowering therapy may retard the progression of diabetic nephropa-

Corresponding author: Dr. R.Bender, Heinrich-Heine-Universität Düsseldorf, Department of Metabolic Diseases and Nutrition, P.O. Box 101007, D-40001 Düsseldorf, Germany thy. However, the statistical analyses and the interpretation of the results give rise to the following criticism.

Firstly, the main hypothesis of any randomized clinical trial regarding the effect of an intervention can only be tested by means of between-group comparisons. However, the main conclusion of Lam et al. [1] was based upon within-group comparisons, which are principally invalid for investigating a treatment effect [2]. All tests between the two groups concerning the main outcome, namely simple $t$-tests for the glomerular filtration rate at each time point, yielded non-significant results. Hence, by means of the statistical analysis used by the authors, an effect of cholesterol-lowering therapy was not demonstrated.

Secondly, for within-group comparisons an invalid statistical method was used, namely two-way analysis of variance. 